\title{
Coconut oil has less satiating properties than medium chain triglyceride oil
}

Article

Accepted Version

Creative Commons: Attribution-Noncommercial-No Derivative Works 4.0

Kinsella, R., Maher, T. and Clegg, M. E. (2017) Coconut oil has less satiating properties than medium chain triglyceride oil. Physiology \& Behavior, 179. pp. 422-426. ISSN 0031-9384 doi: https://doi.org/10.1016/j.physbeh.2017.07.007 Available at https://centaur.reading.ac.uk/80386/

It is advisable to refer to the publisher's version if you intend to cite from the work. See Guidance on citing.

To link to this article DOI: http://dx.doi.org/10.1016/j.physbeh.2017.07.007

Publisher: Elsevier

All outputs in CentAUR are protected by Intellectual Property Rights law, including copyright law. Copyright and IPR is retained by the creators or other copyright holders. Terms and conditions for use of this material are defined in the End User Agreement.

\section{www.reading.ac.uk/centaur}

\section{CentAUR}

Central Archive at the University of Reading

Reading's research outputs online 
1 Coconut oil has less satiating properties than medium chain triglyceride oil

2

3

R. Kinsella T. Maher and M.E. Clegg*

4

Functional Food Centre, Department of Sport and Health Sciences, Faculty of Health and Life

5 Sciences, Oxford Brookes University, Potential mechanisms Gipsy Lane, Oxford OX3 OBP, UK

6

$7 \quad{ }^{*}$ Corresponding author: Miriam Clegg, Functional Food Centre, Department of Sport and

8 Health Sciences, Faculty of Health and Life Sciences, Oxford Brookes University, Gipsy Lane,

9 Oxford OX3 OBP, UK

10 Email: mclegg@brookes.ac.uk; Ph: +44 1865484365

11

12

Running title: MCT, coconut oil and satiety

13

14

15

16

17

18

19

20

21

22

23 
Abstract

It is well established that the consumption of medium-chain triglycerides (MCT) can increase satiety and reduce food intake. Many media articles promote the use of coconut oil for weight loss advocating similar health benefits to that of MCT. The aim of this study was to examine the effect of MCT oil compared to coconut oil and control oil on food intake and satiety. Following an overnight fast, participants consumed a test breakfast smoothie containing $205 \mathrm{kcal}$ of either (i) MCT oil (ii) coconut oil or (iii) vegetable oil (control) on three separate test days. Participants recorded appetite ratings on visual analogue scales and were presented with an ad libitum lunch meal of preselected sandwiches 180 minutes after consumption of the breakfast. The results showed a significant difference in energy and macronutrient intakes at the ad libitum meal between the three oils with the MCT oil reducing food intake compared to the coconut and control oil. Differences in food intake throughout the day were found for energy and fat, with the control having increased food intake compared to the MCT and coconut. The MCT also increased fullness over the three hours after breakfast compared to the control and coconut oils. The coconut oil was also reported as being less palatable than the MCT oil. The results of this study confirm the differences that exist between MCT and coconut oil such that coconut oil cannot be promoted as having similar effects to MCT oil on food intake and satiety.

Keywords: medium chain triglycerides, coconut oil, satiety, food intake 
- It is well established that eating medium-chain triglycerides (MCT) can increase satiety and reduce food intake.

- Many media articles promote the use of coconut oil advocating similar health benefits to that of MCT

- The current study examined the effect of MCT oil compared to coconut oil and and satiety

60

61

62

63

64

65

66

67

68

69

70 


\section{Introduction}

It has been shown previously that high fat diets are linked to the weight gain and potentially obesity, but evidence also suggests that the type of fat consumed and not just the amount of fat is a factor influencing adipose tissue stores [21]. Medium chain triglycerides (MCT) are a type of dietary triglycerides with fatty acids that are 6 to 10 carbon atoms in length [4] and pure MCT oil is manufactured by the hydrolysis, filtering and re-esterification of both palm oil and coconut oil. It has been shown that MCT consumption increases energy expenditure, fat oxidation $[7,11,18]$ and satiety and lowers energy and food intake [14] in both lean and obese individuals. MCT smaller molecular weight allows them to be more rapidly and completely hydrolysed compared to long chain triglycerides (LCT) and can be absorbed when there are decreased intraluminal concentrations of pancreatic enzymes and bile salts [2]. During digestion MCT are converted to medium-chain fatty acids (MCFA) and transported directly in the portal venous system, as opposed to being transported as chylomicrons in the lymphatic system like LCT [1]. MCT therefore bypass peripheral tissues, such as adipose tissue, which makes them less susceptible to the actions of hormonesensitive lipase and to deposition into adipose tissue stores [4]. MCFA can also cross the mitochondrial membrane of the liver and muscle independently of the acylcarnitine transfer system, making them a much more readily available energy source [3].

MCT have been proposed to affect satiety by a number of mechanisms though a lot is still unknown. Potential mechanisms include the anorexigenic effect through the concomitant production of ketones that is a result of increased acetyl-CoA influx which is necessary to oxidize fatty acids $[4,9,15]$. The results of Van Wymelbeke et al. (2001) and Rolls et al. (1988) indicate pre-absorptive mechanisms pertaining to the rapid rate of absorption of 
MCT. Where LCT result in two 'peaks' of absorption; that being at the initial point of ingestion and a second delayed peak at the beginning of the next meal, MCT are fully absorbed at the point of ingestion [10]. Therefore, MCT may increase satiation and satiety immediately after the meal as they are all absorbed in one single bolus rather than being delivered later. However it should be noted that some researchers found that the increase in fat oxidation and postprandial energy expenditure associated with MCFA did not result in any significant differences in ad libitum energy intake or perceived appetite sensations [17].

Many media articles encourage the use of coconut oil for weight loss advocating similar health benefits to that of MCT which has contributed to an increase in consumption of coconut oil in recent years [26]. However MCT oil and coconut oil are not the same. Lauric acid (carbon chain length 12) is found in much larger quantities in coconut oil, making up almost fifty percent of the total fat where no lauric acid is found in MCT oil [26]. Unlike with pure MCT oil containing fatty acids of shorter carbon length (C6-C10) only twenty to thirty percent of lauric acid is taken directly to the liver to be used as energy via the portal vein [8]. Two studies examining the effects of coconut oil compared to LCFAs reported no increase in satiety and no effect on food intake $[23,27]$. Poppit et al [23] found no difference in ratings of satiety or food intake at an ad libitum lunch following eating either coconut oil (containing 10g MCT), high short chain triglycerides (3g SCT, 7g MCT) (from soft fraction milk fat) or long chain triglycerides (from tallow). Rizzo et al [27] found that at a dinner meal following ice-cream containing varying amounts of coconut oil there was trend towards a decreased intake following the coconut oil, however this was compensated for later on when snack consumption increased resulting in no overall difference between the ice-creams. To the best of the authors' knowledge there is a lack of data on the effect of 
coconut oil compared to MCT on food intake and satiety. The aim of this study is to analyse the effect of MCT and coconut oil on food intake and satiety. This study will examine the role that standard MCT and coconut oil play in increasing satiety and reducing food intake over a 24 hour period and will compare them to each other and to a control.

\section{Materials and methods}

127 This is a randomised, single-blind, repeated measures study that fed participants three different test breakfasts on three non-consecutive days.

\subsection{Participants}

131 Twenty eight healthy male and female participants were recruited through personal communication and poster advertisements. Prior to inclusion all participants were given detailed information on the study and were then screened for eating behaviour using the Three-factor eating questionnaire for restrained eating [5] as well as a de-identified health questionnaire detailing any food allergies and/or intolerances; any genetic or metabolic disease; medication and smoking habits. They also had their anthropometric measurements (weight, height, fat percentage) taken using a bio impedance scale (Model BC-418 MA, Tanita UK Ltd., Yiewsley, UK) and freestanding stadiometer (Seca 217, Birmingham, UK). Only participants who did not show signs of restrained eating habits $(<10$ in factor one of the Three Factor eating questionnaire) and satisfied the inclusion criteria were then included in the study. The exclusion criteria were as follows, any metabolic or genetic disease; any medication other than the oral contraceptive pill, any food allergies or intolerances to food included in the study, $\mathrm{BMI}>30 \mathrm{~kg} / \mathrm{m} 2$ and ages outside of 18 and 50 
years. Four participants were excluded from the study at this stage due to being restrained eaters leaving 24 participants that completed the study (table 1 ).

On the day prior to all three test days participants were asked to avoid consumption of caffeine, alcohol and nicotine and refrain from unusual strenuous physical activity that was not part of their normal daily life. The participants were also asked to fast from $9 \mathrm{pm}$ the night before (10-12 hours before testing). Water was allowed. The participants were required to keep a standardised food diary the day prior to the first test day and their diet and physical activity was repeated the day prior to both of the succeeding test days. Researchers provided instructions, scales and food diaries for participants to complete. Ethical approval was granted by the Research Ethics officer in the Department of Sport and Health Sciences in Oxford Brookes University according to the guidelines laid down in the Declaration of Helsinki. Written informed consent was obtained from all participants.

\subsection{Study design}

Participants took part in a randomised, repeated measures, single blind study where they were fed a breakfast high in MCT, coconut oil or a control (vegetable) oil on three nonconsecutive days with at least one day between tests. The minimum number of days between tests was one and the maximum was 14 . Participants had baseline measurements taken and then had fifteen minutes to consume the test breakfast. Following this their satiety and appetite was measured over a period of three hours. 
The test breakfast was $250 \mathrm{ml}$ of a mango and passion fruit smoothie (Tesco stores Ltd, Cheshunt, UK, $143 \mathrm{kcal}$ (606 kJ); 0.3g fat; 31.8g carbohydrates; $1.3 \mathrm{~g}$ protein) with one of the following three lipids: (1) coconut oil (Vita Coco organic extra virgin coconut oil, All Market Europe Ltd, London, UK, 26g (lauric acid 48\%, Caprylic acid 8\% and capric acid 7\%), (2) MCT oil (Muscleform, Norfolk, UK, 25g (caproic acid 2\%, caprylic acid 50-60\%, capric acid $30-45 \%$ and lauric acid 3\%), (3) vegetable oil (rapeseed oil, Tesco stores Ltd, Cheshunt, UK, 23g). The three test oils were isocaloric containing $205 \mathrm{kcal}(858 \mathrm{~kJ})$ and initial pilot testing noted little taste or texture difference between the smoothies. Each test breakfast contained $348 \mathrm{kcal}$ (1456 kJ). The smoothie and fats were mixed for 60 seconds using a food blender and consumed immediately afterwards to avoid oil separation.

\subsection{Subjective satiety and appetite feelings}

Subjective ratings for hunger, fullness, desire to eat and prospective food consumption were recorded using one-hundred-millimetre continuous line visual analogue scales (VAS). Participants completed the VAS before and after consumption of the test breakfast and every 30 minutes for the following 3 hours until they were presented with the ad libitum lunch and the final VAS was completed after they had consumed the lunch.

\subsection{Palatability}

Palatability (how much they liked the drink) was measured directly after consuming the smoothie using a $100 \mathrm{~mm}$ visual analogue scale. 
Three hours after participants consumed their test breakfast they were presented with an ad libitum sandwich lunch. The lunch consisting of sandwiches was given ad-libitum to measure food intake similar to that used by Ranawana et al [24] and Clegg and Thondre [25]. Prior to testing, participants were given a choice of sandwiches from a list and asked to choose which ones they liked. All the sandwich recipes were formulated to contain the same energy content per portion (Table 2). The lunch consisted of three weighed plates each containing two sandwiches cut into quarters. Participants were given all the sandwiches at once so that it was in excess and asked to eat until they felt comfortably full. Participants were given the same sandwiches for each test. The subjects were presented with the meal under identical conditions on each test day. They ate in the same laboratory on their own with no distractions and were given 20 minutes in which to eat their ad libitum meal.

When participants finished eating the remaining food leftover was weighed to measure food intake. A weighed food diary was used to measure food intake for the rest of the day. Volunteers were provided with a food scales and food diary and were given training and instruction on how to complete it. Food diaries were analysed using the software package Nutritics Professional (Est. 2011, Dublin, Ireland).

\subsection{Statistical Analysis}

Statistical analysis was performed using Statistical Package for the Social Sciences (version 23.0; SPSS, Chicago, IL, USA) and data and figures were processed using Microsoft Excel (2006, Reading, UK). A power calculation using actual means and standard deviations from previous satiety research in our laboratory showed that our power to test satiety using VAS AUC was $90 \%$ with 23 participants [25]. 
Data were tested for normality using Shapiro-Wilk test. Following this, a repeated measures ANOVA with pairwise comparisons was used to analyse total food intake and to determine the differences between MCT oil, coconut oil and control oil on food intake during a 24-hour period. The food intake at the ad libitum lunch data and the palatability data were addressed using Friedman's test due to a non-normal distribution. Wilcoxon signed Rank test was used to determined individual differences between MCT oil, coconut oil and control oil on food intake during the ad libitum lunch. For the VAS, the areas under the curves (AUC) were calculated using the trapezoidal rule. The data was analysed using an ANOVA, with the baseline value as a covariate in the analysis (Blundell et al., 2010). Values are presented as means \pm standard deviation. The significance value was set at $p<0.05$.

\section{Results}

\subsection{Food intake at the ad libitum lunch}

For the ad libitum lunch there were significant differences in the mass of food consumed $(\chi 2(2)=9.083, p=0.011)$, energy $(\chi 2(2)=7.583 p=0.023)$, carbohydrate $(\chi 2(2)=7.750$, $p=0.021)$, protein $(\chi 2(2)=9.083, p=0.011)$ and fat $(\chi 2(2)=9.000, p=0.011)$ intake between the three smoothies. The differences were between the control and MCT and between the MCT and coconut oil such that the MCT oil reduced food intake at the ad libitum lunch more than the other two oils (table 3).

\subsection{Total food intake throughout the day}

There were significant differences in energy intake $(F(2)=4.548, p=0.016)$ and fat consumption $(F(2)=4.659, p=0.14)$ throughout the day between the three oils (table 3 ). 
There were no significant differences in carbohydrate and protein intakes for the entire day between the three oils tested.

The differences in energy intake were between the control oil and the MCT oil $(\mathrm{t}(23)=$ 2.571, $p=0.017)$ and between the control oil and the coconut oil $(t(23)=2.124, p=0.045)$. The highest energy intake was consumed after the breakfast containing the control oil, an average of $428 \mathrm{Kcal}(1796 \mathrm{~kJ})$ extra were consumed compared with the breakfast containing the MCT oil and an extra $280 \mathrm{kcal}(1180 \mathrm{~kJ})$ was consumed following the control oil compared to the coconut oil. There was no significant difference between energy intake after the consumption of coconut oil and MCT oil.

The significant differences found for fat consumption were between the control oil and the MCT oil $(t(23)=2.607, p=0.016)$. An extra $14 \mathrm{~g}$ of fat was consumed after the control oil compared to the MCT oil. There were no significance differences for fat intake between control and coconut oil or between MCT and coconut oil.

\subsection{Perceived satiety}

There were no significant differences for three of the four satiety parameters that were measured using the VAS: hunger, desire to eat and prospective food consumption ( $p>0.05)$. There were significance differences for the fullness parameter $(F(2)=3.427, p=0.038)$, these differences existed between the control and MCT oil $(p=0.021)$ and between the MCT and coconut oil ( $p=0.037)$ (Figure 1). The highest perception of fullness was found after the consumption of MCT oil compared with control and coconut oil. No differences were found for fullness between control oil and coconut oil. In all tests the feelings of satiety increased 
following the breakfast and then gradually decreased until the ad libitum buffet (Figure 2ad).

3.4 Palatability

There was a difference in palatability between the three smoothies (control: $72.3 \pm 18.7$;

MCT: $73.0 \pm 23.1$; coconut: $63.9 \pm 22.8 ; \chi 2(2)=6.156, p=0.046)$, the difference was between MCT and coconut oil $(\mathrm{Z}=-2.221, \mathrm{p}=0.026)$. The $\mathrm{MCT}$ was recorded as being more palatable than the coconut oil.

\section{Discussion}

To the best knowledge of the authors this is the first study to compare the effects of MCT and coconut oil against each other and to a control LCFA for satiety and food intake. Studies have previously shown that MCT demonstrates beneficial effects by increasing satiety and reducing food intake over a period of a day $[6,13,16]$ and this was confirmed in the current study where the MCT oil reduced food intake both at the ad libitum meal and throughout the day compared to a control LCFA oil. Differences in food intake following coconut oil are not as well documented despite much media speculation in relation to their satiating properties [26].

In the current study, the coconut oil did not reduce food intake at the ad libitum meal. There were, however significant differences in food intake throughout the day with the coconut oil reducing food intake compared to the LCFA oil though not to the same extent as the MCT oil. Given that the coconut oil contains significantly less MCT and that the MCT has mostly caused the increase in satiety, this is not a particularly surprising effect. It highlights 
that the distinction between the two oils needs to be made especially in the media. Previous research on the effect of coconut oil is limited however two studies have been completed. Research from Poppitt et al [23] found a lack of difference in visual analogue scale ratings of satiety or ad libitum food intake between dairy fats (MCT and short chain fatty acids), coconut oil and beef tallow (saturated long chain fatty acids). In a later study by Rizzo et al. [27] they found that coconut oil did reduce fat intake and there was a trend towards a reduction in energy intake at an ad libitum meal following a high coconut oil ice cream. However this appeared to be compensated for later in the day. It should also be noted that amounts of lipids given in this study were over half that given in the current study.

The lack of similarity between MCT and coconut oil results may be due to their structure. Coconut oil is a natural source of MCFAs oils and the main MCFA that makes up coconut oil is lauric acid ( $50 \%$ ) [8], while MCT oil has a lower amount of lauric acid (1-3\%) [4]. Lauric acid has a chain length 12 carbons and due this it's metabolism can differ to that of MCT oil (caproic fatty acids (C6:0), caprylic fatty acid (C8:0), capric fatty acid (C10:0)) [20]. Some authors such as Denke \& Grundly [8] affirm that only $20-30 \%$ of lauric acid is absorbed by the portal vein directly to the liver and the rest of lauric acid is absorbed using chylomicrons like LCFAs do [8]. These warrants further research into the metabolism of lauric acid and the similarity to the metabolism of the rest of MCFAs. It should also be noted that overall combination of lauric, caprylic and capric acid present in the coconut oil was only $63 \%$ compared with the remainder being LCFA. The MCT oil consisted of all MCFA. 
Nausea was not measured during the trial however feelings of nausea were reported by five of the participants of the study after having the MCT oil, while no side effects were reported after the consumption of either the coconut or control oil. These could have affected the participant's food intake and the VAS scores. It has been demonstrated that MCT can cause side effects including stomach cramping and nausea [22] however it has previously always been associated with quite high doses of $\sim 85 \mathrm{~g}$ given in exercise studies [12]. This shows that even a dose as small as $25 \mathrm{~g}$ of MCT can have side effects which may have impacted in their food intake. Nonetheless it was the coconut oil smoothies that were found to be the least palatable. This is in contradiction to the hypothesis that MCFA have a repulsive taste and MCT may be broken down into MCFA by lingual lipase early on in digestion causing people to eat less $[4,6]$, however given it was a smoothie it was unlikely to remain in the mouth for a prolonged period for any reasonable digestion to occur. The dislike of the coconut beverage could potentially have been due to participants disliking the taste of coconut, however this was quite strongly masked by the smoothie drink, as was found in our pilot testing.

There are several limitations to this study. The study excluded obese individuals. This decision was made as is has been shown that MCT may potentially be less effective in obese individuals $[11,19]$, however this area does warrant further research. The study also used a high dose of fat, and consuming $25 \mathrm{~g} \mathrm{MCT}$ in a single setting would not be pragmatic or recommended, however it was based on similar studies that had shown positive satiating effects of MCT $[13,16]$. Future studies should address this by using smaller doses that are more representative of single meals. Participants were aware that their food intake was being measured, however none commented on noticing any differences between the three 
smoothies so were unlikely to behave differently based on this. Finally female participants were not tested at the same phase of their menstrual cycle.

\section{Conclusion}

Overall the research indicates that the effects seen in for MCT oil are not the same as those found for coconut oil, however given that the coconut oil contains less MCT this is not surprising. The coconut oil given in the current study did reduce food intake throughout the day, however it must be remembered that this was given in a dose of $26 \mathrm{~g}$ which is likely to be more than an individual would generally consume in one day. Further research is needed using smaller doses of coconut oil in obese and overweight individuals.

Acknowledgements: This study was funded by Oxford Brookes University. We thank Noelia Hernandez Cuidad for her help with data collection. 
357 1. Bloom, B., I.L. Chaikoff, and Reinhardt, Intestinal lymph as pathway for transport of absorbed fatty acids of different chain lengths. Am J Physiol, 1951. 166(2): p. 451-5.

2. Fernandes, J., K.J. van de, and H.A. Weijers, Differences in absorption of the various fatty acids studied in children with steatorrhea. J Clin Invest, 1962. 41: p. 488-94.

3. Williamson, J.R., et al., Inhibition of fatty acid stimulation of gluconeogenesis by (+)-

4. Bach, A.C. and V.K. Babayan, Medium-chain triglycerides: an update. Am J Clin Nutr, 1982. 36(5): p. 950-62.

5. Stunkard, A.J. and S. Messick, The three-factor eating questionnaire to measure

6. Rolls, B.J., et al., Food intake in dieters and nondieters after a liquid meal containing

8. Denke, M.A. and S.M. Grundy, Comparison of effects of lauric acid and palmitic acid on plasma lipids and lipoproteins. Am J Clin Nutr, 1992. 56(5): p. 895-8.

9. Bach, A.C., Y. Ingenbleek, and A. Frey, The usefulness of dietary medium-chain triglycerides in body weight control: fact or fancy? J Lipid Res, 1996. 37(4): p. 708-26.

10. Fielding, B.A., et al., Postprandial lipemia: the origin of an early peak studied by specific dietary fatty acid intake during sequential meals. Am J Clin Nutr, 1996. 63(1): p. 36-41. 
379 11. Binnert, C., et al., Influence of human obesity on the metabolic fate of dietary longand medium-chain triacylglycerols. Am J Clin Nutr, 1998. 67(4): p. 595-601.

381

382

12. Jeukendrup, A.E., et al., Effect of medium-chain triacylglycerol and carbohydrate ingestion during exercise on substrate utilization and subsequent cycling performance. Am J Clin Nutr, 1998. 67(3): p. 397-404.

13. Van Wymelbeke, V., et al., Influence of medium-chain and long-chain triacylglycerols on the control of food intake in men. Am J Clin Nutr, 1998. 68(2): p. 226-34.

14. Krotkiewski, M., Value of VLCD supplementation with medium chain triglycerides. Int J Obes Relat Metab Disord, 2001. 25(9): p. 1393-400.

15. Tsuji, H., et al., Dietary medium-chain triacylglycerols suppress accumulation of body fat in a double-blind, controlled trial in healthy men and women. J Nutr, 2001. 131(11): p. 2853-9.

16. Van Wymelbeke, V., J. Louis-Sylvestre, and M. Fantino, Substrate oxidation and control of food intake in men after a fat-substitute meal compared with meals supplemented with an isoenergetic load of carbohydrate, long-chain triacylglycerols, or medium-chain triacylglycerols. Am J Clin Nutr, 2001. 74(5): p. 620-30.

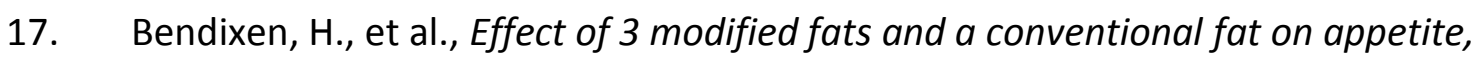
energy intake, energy expenditure, and substrate oxidation in healthy men. Am J Clin Nutr, 2002. 75(1): p. 47-56.

18. St-Onge, M.P., et al., Medium-versus long-chain triglycerides for 27 days increases fat oxidation and energy expenditure without resulting in changes in body composition in overweight women. Int J Obes Relat Metab Disord, 2003. 27(1): p. 95102. 
402

403

404

405

406

407

408

409

410

411

412

413

414

415

416

417

418

419

420

421

422

423

424

19. St-Onge, M.P. and P.J. Jones, Greater rise in fat oxidation with medium-chain triglyceride consumption relative to long-chain triglyceride is associated with lower initial body weight and greater loss of subcutaneous adipose tissue. Int J Obes Relat Metab Disord, 2003. 27(12): p. 1565-71.

20. Marten, B., M. Pfeuffer, and J. Schrezenmeir, Medium-chain triglycerides. International Dairy Journal, 2006. 16(11): p. 1374-1382.

21. Fernandez-Quintela, A., I. Churruca, and M.P. Portillo, The role of dietary fat in adipose tissue metabolism. Public Health Nutr, 2007. 10(10A): p. 1126-31.

22. Clegg, M.E., Medium-chain triglycerides are advantageous in promoting weight loss although not beneficial to exercise performance. Int J Food Sci Nutr, 2010.

23. Poppitt, S.D., et al., Fatty acid chain length, postprandial satiety and food intake in lean men. Physiol Behav, 2010. 101(1): p. 161-7.

24. Ranawana, V., A. Muller, and C.J. Henry, Polydextrose: its impact on short-term food intake and subjective feelings of satiety in males-a randomized controlled cross-over study. Eur J Nutr, 2013. 52(3): p. 885-93.

25. Clegg, M.E. and P.S. Thondre, Molecular weight of barley beta-glucan does not influence satiety or energy intake in healthy male subjects. Appetite, 2014. 83: p. 167-72.

26. Lockyer, S. and S. Stanner, Coconut oil - a nutty idea? Nutrition Bulletin, 2016. 41: p. 42-54.

27. Rizzo, G., et al., Coconut and sunflower oil ratios in ice cream influence subsequent food selection and intake. Physiol Behav, 2016. 164(Pt A): p. 40-46. 
427 Table 1: Participant characteristics

\begin{tabular}{llll}
\hline & Female & Male & Both \\
& $(\mathrm{n}=18)$ & $(\mathrm{n}=6)$ & $(\mathrm{n}=24)$ \\
\hline Age (years) & $28.1 \pm 6.6$ & $24.8 \pm 2.7$ & $27.5 \pm 6.0$ \\
Height $(\mathrm{m})$ & $1.66 \pm 0.07$ & $1.74 \pm 0.05$ & $1.68 \pm 0.07$ \\
Weight $(\mathrm{kg})$ & $62.0 \pm 7.4$ & $70.1 \pm 9.7$ & $64.5 \pm 8.5$ \\
BMI (kg.m $\left.{ }^{2}\right)$ & $22.6 \pm 2.5$ & $23.2 \pm 2.3$ & $22.9 \pm 2.4$
\end{tabular}

428

429

430

431

432

433

434

435

436

437

438

439

440

441

442 
444 Table 2: Nutritional content of sandwiches (ad libitum lunch)

Sandwich: $\quad$ Weight $(\mathrm{g}) \quad$ Energy $(\mathrm{kcal}(\mathrm{kJ})) \quad$ Carbohydrate $(\mathrm{g}) \quad$ Protein $(\mathrm{g}) \quad$ Fat $(\mathrm{g})$

\begin{tabular}{llllll}
\hline Egg mayo & 223 & $408.20(1709)$ & 36.68 & 17.46 & 19.81
\end{tabular}

Cheese and

tomato

185

$406.06(1700) \quad 36.62$

19.73

18.51

Tuna mayo 146

$402.79(1686) \quad 35.30$

18.37

19.56

Chicken salad 221

$406.48(1701)$

37.51

18.61

18.66

Cheese and

pickle

148

404.75 (1695)

38.98

19.03

17.75

Ham and

cheese

153

405.43 (1698)

35.62

21.49

18.21

Roast beef

and tomato

181

404.30 (1693)

36.55

20.02

18.11

445

446

447

448

449

450

451

452

453

454 
Table 3: Energy and macronutrient intake at the ad libitum lunch and the day's total intake

\begin{tabular}{|c|c|c|c|}
\hline & Control & MCT & Coconut \\
\hline \multicolumn{4}{|c|}{ Ad libitum lunch } \\
\hline Energy (kcal) & $1680 \pm 498$ & $1438 \pm 573^{*}$ & $1612 \pm 502^{+}$ \\
\hline kJ & $7023 \pm 2084$ & $6011 \pm 2397^{*}$ & $6738 \pm 2099^{+}$ \\
\hline Carbohydrate (g) & $155 \pm 47$ & $132 \pm 54^{*}$ & $149 \pm 47^{+}$ \\
\hline Protein (g) & $78 \pm 24$ & $67 \pm 27^{*}$ & $75 \pm 23^{+}$ \\
\hline Fat (g) & $77 \pm 22$ & $66 \pm 26^{*}$ & $74 \pm 23^{+}$ \\
\hline \multicolumn{4}{|c|}{ Total day intake } \\
\hline Energy (kcal) & $2992 \pm 714$ & $2564 \pm 918^{*}$ & $2712 \pm 546^{*}$ \\
\hline kJ & $12518 \pm 2995$ & $10722 \pm 3841^{*}$ & $11338 \pm 2284^{*}$ \\
\hline Carbohydrate (g) & $295 \pm 69$ & $261 \pm 110$ & $269 \pm 62$ \\
\hline Protein (g) & $142 \pm 43$ & $125 \pm 49$ & $131 \pm 33$ \\
\hline Fat (g) & $132 \pm 36$ & $108 \pm 37^{*}$ & $118 \pm 27$ \\
\hline
\end{tabular}

457

458

$* p<0.05$ compared to control

459

${ }^{+} \mathrm{p}<0.05$ compared to MCT

460

461

462

463

464 
466

467

468

469

470

471

472

473

474

475

476

477

478

479

480

481

482

483

Figure headings

Figure 1: Area under the curve for hunger, fullness, desire to eat and prospective

consumption following the breakfast containing either control oil, MCT oil or coconut oil.

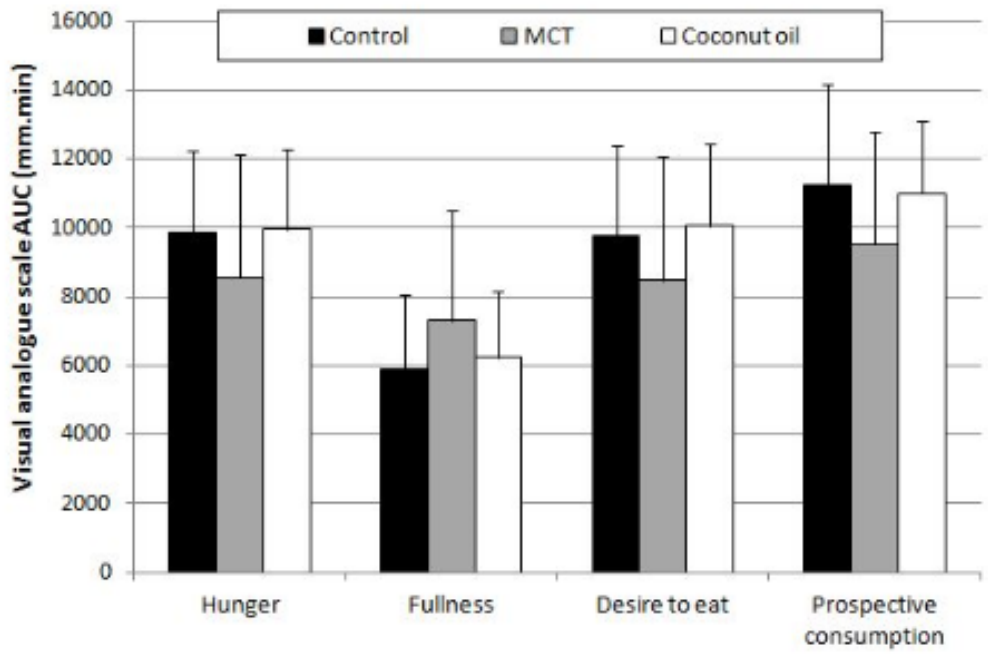

\section{(1)}

2


484

485

486

487

488

489

490

491

492

493

494

495

496
Figure 2: Visual analogue scale data for hunger, fullness, desire to eat and prospective consumption at baseline ( $0 \mathrm{~min}$ ), between the breakfast (of either control oil, MCT oil or coconut oil) and the ad libitum meal and after the ad libitum meal
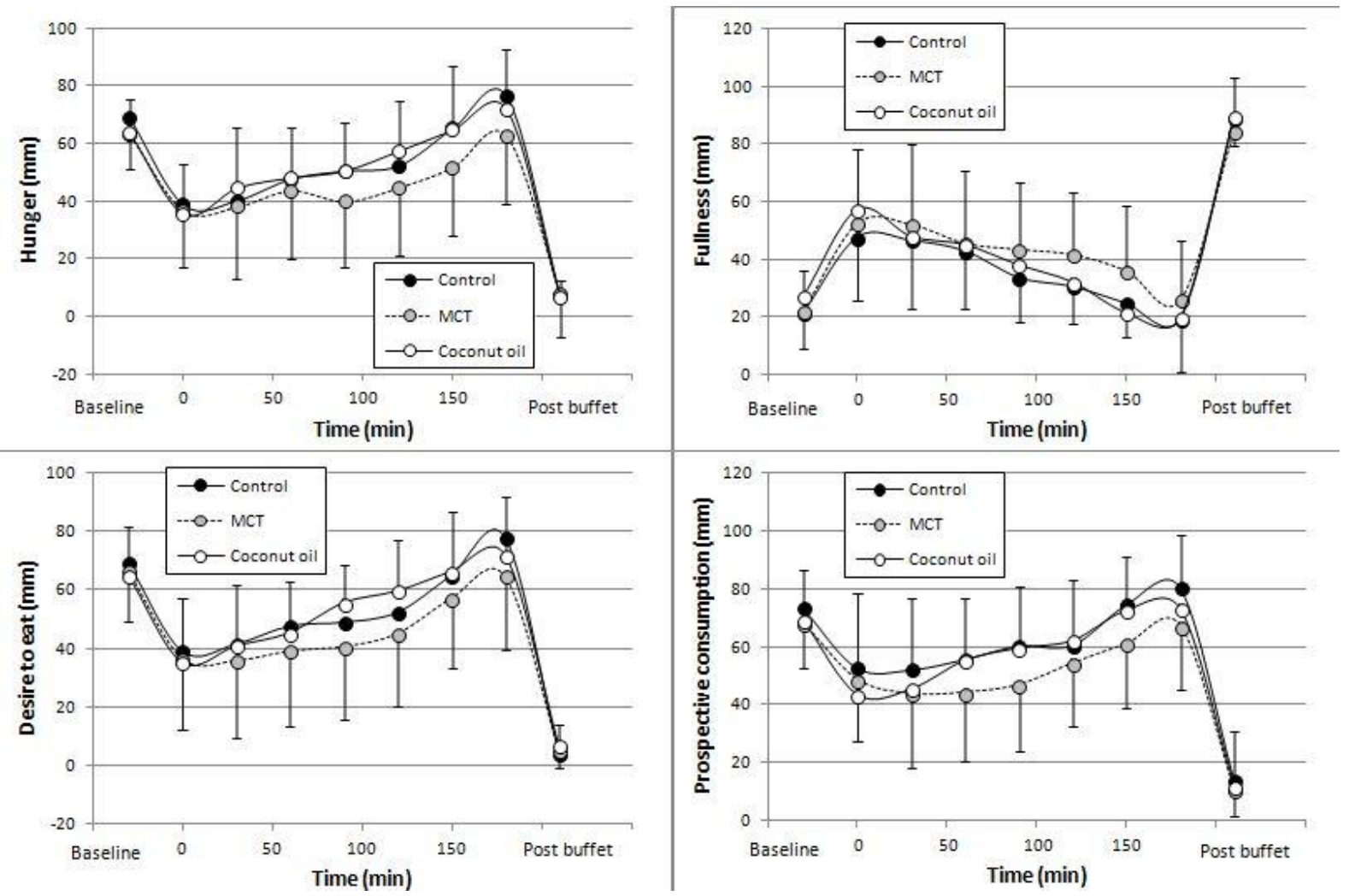